\title{
Preliminary Report: Asymmetric Crypt Fission in Biopsies from Patients With Ulcerative Colitis
}

\author{
CARLOS A. RUBIO ${ }^{1}$ and PETER T. SCHMIDT ${ }^{2}$ \\ ${ }^{1}$ Gastrointestinal and Liver Research Laboratory, Department of Pathology, Karolinska \\ Institute and University Hospital, Centre for Digestive Diseases, Stockholm, Sweden; \\ ${ }^{2}$ Department of Medicine, Karolinska Institute and University Hospital, \\ Centre for Digestive Diseases, Stockholm, Sweden
}

\begin{abstract}
Background: Recently, we found crypts with asymmetric fission bordering ulcers in colectomy specimens from patients with ulcerative colitis $(U C)$. Here, we report crypts with asymmetric fission found in biopsies from patients with UC. Patients and Methods: Sections from endoscopic biopsies from five patients with UC were reviewed. The number of transected (cut-across) crypts in symmetric and asymmetric fission was assessed in sections from three biopsies in each patient. Results: A total of 89 crypts in fission were recorded in the 15 biopsies; 36 $(40.4 \%)$ were in symmetric fission and the remaining 53 (59.6\%) in asymmetric fission. Conclusion: A high frequency of asymmetric crypts in fission was demonstrated in endoscopic biopsies from patients with UC. It is suggested that this previously unaddressed histological parameter is included in pathological descriptions of endoscopic biopsies from patients with UC.
\end{abstract}

The mucosa of the normal colon is histologically built of close mucosal invaginations or crypts, arranged perpendicular to the surface epithelium and to the muscularis mucosae $(1,2)$. The building of new crypts is accomplished by crypt bifurcations, known as symmetric fissions; beginning at the base of the normal crypts, fission progresses upwards until two identical crypts are finally formed (3). Notably, crypt fission in the normal colon mucosa rarely occurs during adult life $(1,2)$.

This article is freely accessible online.

Correspondence to: Carlos A. Rubio, MD, Ph.D., Gastrointestinal and Pathology Research Laboratory, Department of Pathology, Karolinska Institute and University Hospital, 17176, Stockholm, Sweden. Tel: +46 851774527, Fax: +46 851774524, e-mail: Carlos.Rubio@ki.se

Key Words: Colon, crypt fission, asymmetric fission, morphology.
In 1972, Morson found crypt budding and crypt branching in rectal biopsies from patients with ulcerative colitis (UC) in remission (4). In 1984, we analyzed rectal biopsies from 61 patients with UC in remission (5). Biopsies having transversally cut glands exhibited lateral crypt buddings and dichotomy crypts. Dichotomy of the glands was easily recognized at the point of bifurcation; it was called the back-to-back sign, a visual metaphore for crypts in fission (5).

More recently, Tan et al. found that about $90 \%$ of dissected single colonic crypts from C57BL/6 mice replicated by 'buds' growing near the bottom in individual crypts; this phenomenon was called asymmetric bud fission (6). On the other hand, Bomman and Fields claimed that only dysplastic-adenomatous colonic crypts develop buddings and bifurcations (7).

In previous work, in sections from three colectomies with UC, we found that $43 \%$ of the crypts with corrupted shapes bordering ulcers exhibited asymmetric fission (8). In a more recent review of sections from seven colectomy specimens with UC, we found that out of 3,586 crypts in fission, $82 \%$ were in asymmetric fission and only $18 \%$ in symmetric fission (9). The conclusion was that asymmetrical crypt fission is a common finding in colectomy specimens from patients with active UC.

The purpose of this communication is to report and illustrate recent observations revealing that crypts with asymmetric fission are also found in routine biopsies from patients with active UC.

\section{Patients and Methods}

Three endoscopic biopsies from the left colon were investigated in each of three patients with UC. Hematoxylin and eosin (H\&E)stained sections were scrutinized, and searched for crypts with symmetric and with asymmetric fission.

Most sections from endoscopic biopsies often show transected (cut-across) crypts. Therefore, for comparative purposes, the counting was performed on transected crypts. 


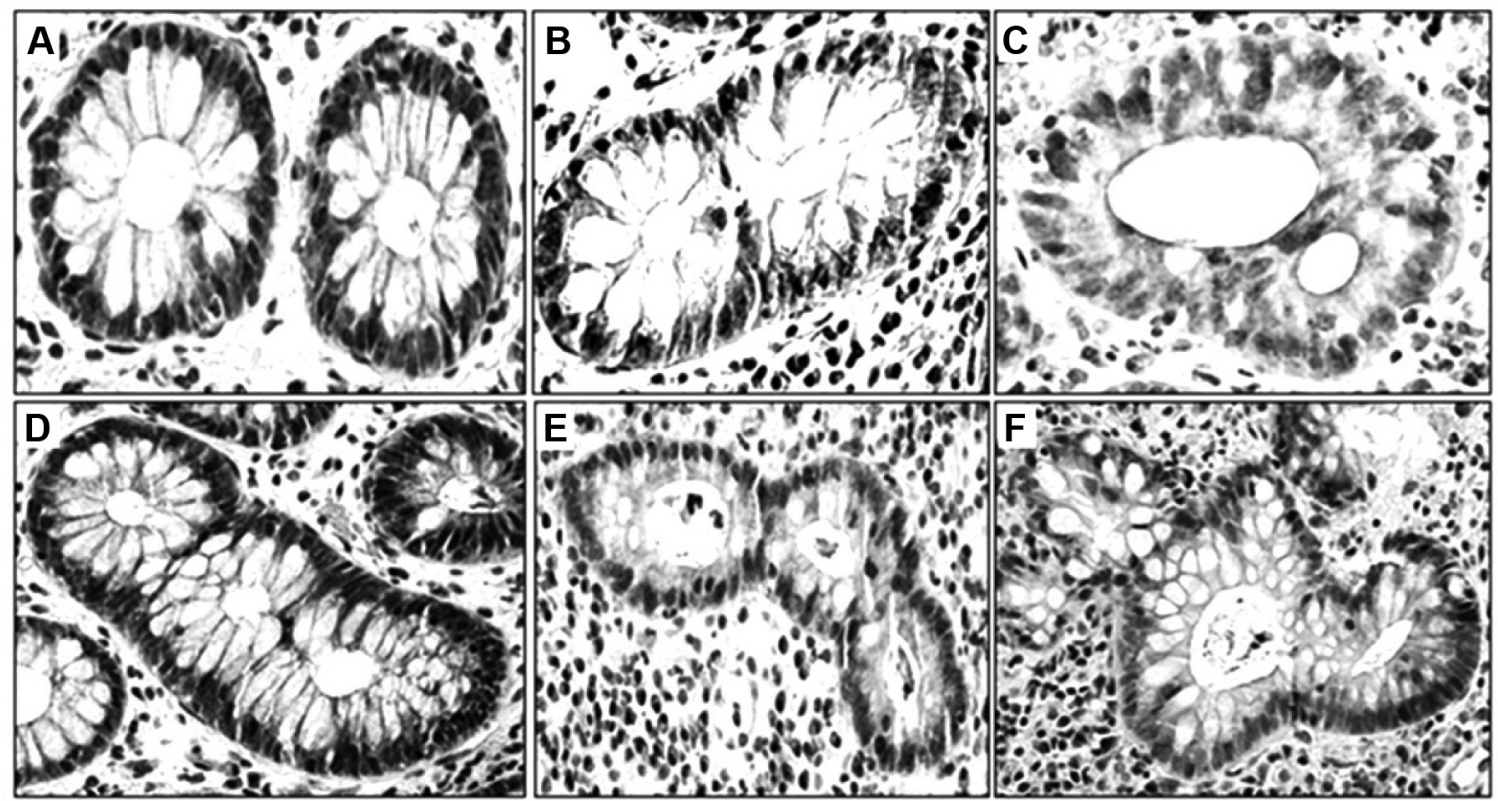

Figure 1. Colonic crypts found in endoscopic biopsies in patients with protracted-active ulcerative colitis stained with hematoxylin and eosin (original magnification). A: Two normal crypts separated by a rim of lamina propria $(\times 20)$. B: Crypt in symmetric fission separated by epithelial rim (x10). C: Crypt in asymmetric fission separated by epithelial rim $(\times 20)$. D: Crypt in trefoil asymmetric fission $(\times 10)$. E: Another crypt in trefoil asymmetric fission $(\times 10) . F$ : Crypt in trefoil asymmetric fission showing extreme asymmetry $(\times 10)$.

Definitions. Crypts without fission: The majority of crypts are not in fission; they appear as "rings" separated from each other by lamina propria (Figure 1A).

Crypts with symmetric fission: Two back-to-back identical crypts, separated by an epithelial rim.

Crypts with asymmetric fission: Two or more back-to-back crypts with asymmetric fission, separated by an epithelial rim.

The number of transected (cut-across) crypts with symmetric and asymmetric fission was assessed in sections from three biopsies. Counting was carried out using a standard Nikon light microscope (Nikon, Surbiton, UK), carrying $\times 10$ ocular lenses and a $\times 10$ Plan Apo objective (aperture 0.45).

The Regional Ethical Review Board in Stockholm approved this study (EPM dnr 2020-01634).

\section{Results}

The results summarized in Table I show that a total of 89 crypts in fission were recorded in the 15 biopsies; 36 $(40.4 \%)$ were in symmetric fission (Figure 1B) and the remaining $53(59.6 \%)$ in asymmetric fission (Figure $1 \mathrm{C}-\mathrm{F}$ ).

The mean number of symmetric crypts per biopsy was 7.2 (range=1-4), and the mean number of asymmetric crypts in fission per biopsy was 10.6 (range $=1-6)$.

\section{Discussion}

In this preliminary report, we showed that crypts with symmetric and with asymmetric fission do occur in
Table I. The number of asymmetrical and symmetrical crypt fission in three endoscopic biopsies/case in five patients with ulcerative colitis.

\begin{tabular}{|c|c|c|c|c|}
\hline \multirow[b]{2}{*}{ Case } & & \multicolumn{3}{|c|}{ Number of crypts with fission } \\
\hline & & Symmetric & Asymmetric & Total \\
\hline \multirow[t]{2}{*}{1} & Total & 7 & 11 & 18 \\
\hline & Mean (range) & $2.3(2-3)$ & $3.7(2-5)$ & $6.0(3-8)$ \\
\hline \multirow[t]{2}{*}{2} & Total & 9 & 13 & 22 \\
\hline & Mean (range) & $3.0(2-4)$ & $4.3(2-6)$ & $7.3(2-6)$ \\
\hline \multirow[t]{2}{*}{3} & Total & 8 & 12 & 20 \\
\hline & Mean (range) & $2.7(2-4)$ & $4.0(2-6)$ & $6.7(2-6)$ \\
\hline \multirow[t]{2}{*}{4} & Total & 7 & 10 & 17 \\
\hline & Mean (range) & $2.3(2-3)$ & $3.3(2-5)$ & $5.7(2-5)$ \\
\hline \multirow[t]{2}{*}{5} & Total & 5 & 7 & 12 \\
\hline & Mean (range) & $1.7(1-2)$ & $2.3(1-4)$ & $4.0(1-4)$ \\
\hline \multirow[t]{2}{*}{ All } & Total & 36 & 53 & 89 \\
\hline & Mean (range) & $7.2(1-4)$ & $10.6(1-6)$ & $17.8(1-8)$ \\
\hline
\end{tabular}

endoscopic biopsies from patients with UC. Importantly, $60 \%$ of the crypts in fission were asymmetric.

One pertinent question is which molecular signals choreograph normal crypt fission? WNT signaling regulates key events during embryonic patterning and morphogenesis (10). In colonic crypts, WNT signaling controls cell proliferation, differentiation and apoptosis. WNT concentration is high at the crypt bottom (where stem cells 
reside), and low at the top of the crypt. In contrast, the concentration of adenomatous polyposis coli (APC) is low at the crypt bottom and high at the top (where differentiated cells reside) $(3,7)$. WNT signaling has been linked to crypt fission in infant rats, and is required for the production of colonic crypts in mice in vitro (3). Thus, normal crypt fission is triggered by WNT signaling.

Another pertinent question is which molecular signals orchestrate the formation of asymmetric crypts in UC? Thirty-six years ago, we reported the presence of crypt dichotomy at the point of crypt bifurcation (i.e. fission) in biopsies from patients with UC in remission; we named it the back-to-back sign (5).

In 1998, Wasan et al. micro-dissected individual crypts from patients with familial adenomatous polyposis (FAP) (3). Single crypts with small epithelial buds, termed asymmetrical bud fission, were more frequently seen in patients with FAP than in controls. Years later, Tan et al. isolated crypts from the colon in C57BL/6 mice; they found that about $90 \%$ of the crypts near the bottom had asymmetrical buds (6). In contrast, Bomman and Fields claimed that colonic crypts developed buddings and bifurcations only after they became dysplastic, i.e. during the formation of adenomas (7). Jagan et al. found that the formation of normal colorectal crypts was regulated by phosphatase and tensin homolog deleted on chromosome, a protein encoded by the PTEN gene (11). Subsequently, Georgescu et al. found that NHERF1 protein, a $\mathrm{Na}^{+} / \mathrm{H}^{+}$ exchanger regulatory factor, controls human colonic gland morphogenesis (12). From the above, it is not inconceivable that the development of crypts with asymmetric fission in patients with UC might be conveyed via mutations of the WNT signaling, and/or by mutations of NHERF1 or PTEN signaling.

More research is necessary to ascertain whether putative mutated asymmetric crypt fissions have any bearing on the predisposition of colonic crypts to develop epithelial dysplasia in patients with UC (13). It is suggested that this previously unaddressed histological parameter is included in pathological descriptions of biopsies from patients with UC.

\section{Conflicts of Interest}

None to declare.

\section{Authors' Contributions}

CAR: Conceptualization, investigation, writing-original draft, illustrations. PTS: Harvested endoscopic biopsies, reviewed the manuscript and added valuable suggestions.

\section{References}

1 Levine DS and Haggitt RC: Normal histology of the colon. Am J Surg Pathol 3: 966-984, 1989. PMID: 2679155.
2 Rubio CA: The histologic structure of the large bowel mucosa and the evolution of the three pathways of colonic carcinogenesis in humans and in experimental animals. In: Recent Studies on Digestive System Anatomy. Lachter I, Uchima H, Tiwarir S and Hamid A (eds.). Chapter 3, pp. 1-12, 2018. Available at: https://openaccessebooks.com/digestive-system-anatomy/Thehistologic-structure-of-the-large-bowel-mucosa-and-the-evolutionof-the-three-pathways-of-colonic-carcinogenesis-in-humans-andin-experiment.pdf [Last accessed on May 6th, 2020]

3 Wasan H S, Park H S, Liu K C, Mandir N K , Winnett A, Sasieni P, Bodmer WF, Goodlad RA and Wright NA: APC in the regulation of intestinal crypt fission J Pathology 185: 246-255, 1998. PMID: 9771477. DOI: 10.1002/(SICI)1096-9896(199807) 185:3<246::AID-PATH90>3.0.CO;2-8

4 Morson BC: Rectal biopsy in inflammatory bowel disease $\mathrm{N}$ Engl J Med 287: 1337-1339, 1972. PMID: 4564312. DOI: 10.1056/NEJM197212282872607

5 Rubio CA, Johansson C, Uribe A and Kock Y: A quantitative method of estimating inflammation in the rectal mucosa. IV. Ulcerative colitis in remission. Scand J Gastroenterol 19: 525530, 1984. PMID: 6463576.

6 Tan CW, Hirokawa Y, Gardiner B, Smith DW and Burgess AW: Colon cryptogenesis: Asymmetric budding. PLoS One 8: e78519, 2013. PMID: 24205248. DOI: 10.1371/journal. pone. 0078519

7 Boman MB and Fields JZ: An APC:WNT counter-current-like mechanism regulates cell division along the human colonic crypt axis: A mechanism that explains how APC mutations induce proliferative abnormalities that drive colon cancer development. Front Oncol 3: 244-254, 2013. PMID: 24224156. DOI: 10.3389/fonc. 2013.00244

8 Rubio CA: Corrupted colonic crypts bordering regenerating mucosal ulcers in ulcerative colitis. In Vivo 31: 669-671, 2017. PMID: 28652436. DOI: 10.21873/invivo.11110

9 Rubio CA and Schmidt PT: Asymmetric crypt fission in colectomy specimens from patients with IBD. J Clin Pathol submitted, 2020; jclinpath-2020-206694

10 Komiya Y and Habas R: WNT signal transduction pathways. Organogenesis 4: 68-75, 2008. PMID: 19279717. DOI: 10.4161/org.4.2.5851

11 Jagan IC, Deevi RK, Fatehullah A, Topley R, Eves J, Stevenson $\mathrm{M}$, Loughrey $\mathrm{M}$, Arthur $\mathrm{K}$ and Campbell FC: PTEN phosphatase-independent maintenance of glandular morphology in a predictive colorectal cancer model system. Neoplasia 15: 1218-1230, 2013. PMID: 24348097. DOI: 10.1593/neo.121516

12 Georgescu MM, Cote G, Agarwal NK and White CL: NHERF1/EBP50 controls morphogenesis of 3D colonic glands by stabilizing PTEN and ezrin-radixin-moesin proteins at the apical membrane. Neoplasia 16: 365-374, 2014. PMID: 24862762. DOI: 10.1016/j.neo.2014.04.004

13 Leedham SJ, Graham TA, Oukrif D, McDonald SA, RodriguezJusto M, Harrison RF, Shepherd NA, Novelli MR, Jankowski JA and Wright NA: Clonality, founder mutations, and field cancerization in human ulcerative colitis-associated neoplasia. Gastroenterology 136: 542-550, 2009. PMID: 19103203. DOI: $10.1053 /$ j.gastro .2008 .10 .086

Received April 22, 2020

Revised May 8, 2020

Accepted May 11, 2020 